\title{
Versorgungsmanagement-
}

Pauschale als Grundlage für

die Weiterentwicklung des

Gesundheitssystems

Die Gesundheitspolitik der CDU, CSU und FDP steht für „Mehr Wettbewerb, mehr Transparenz und weniger Bürokratie“. Jedoch ist der Anreiz im System weiter beschränkt auf Kostendämpfungsinterventionen. Innovationsvermeidung und fehlende Berücksichtigung von Patientenpräferenzen sind die Konsequenz. Die Weiterentwicklung der DMPProgrammkostenpauschale bietet die Chance auf Prozessinnovationen durch Selektivverträge. Das nächste Gesetz zur Reform der medizinischen Versorgung sollte die finanzielle Grundlage für ein nachhaltiges Versorgungsmanagement, das nach einheitlichen Kriterien gesundheitsökonomisch evaluiert wird, bilden.

\section{Heinz Giesen}

\section{Versorgungsmanagement als kritischer Erfolgsfaktor im Kassenwettbewerb}

„Mehr Wettbewerb, mehr Freiheit, weniger Bürokratie“ - das ist die zentrale Forderung des Gesundheitsministers für die Gesundheitspolitik dieser Legislaturperiode ${ }^{1}$. Krankenkassen stehen im Wettbewerb um eine bestmögliche effektive und effiziente Gesundheitsversorgung. Dass es noch viel Optimierungspotenzial gibt, ist in einer Vielzahl an Publikationen beschrieben ${ }^{2}$. Der Wettbewerb der Krankenkassen gestaltet sich nun zunehmend über die neuen Versorgungsformen ${ }^{3}$.

Nachdem mit dem GKV-Modernisierungsgesetz (GMG) im Jahr 2003 Hausarztverträge erstmalig verpflichtend anzubieten waren, wurde mit dem GKV-Wettbewerbsstärkungsgesetz (GKV-WSG) im Jahr 2007 und dem GKVOrganisationsweiterentwicklungsgesetz (GKV-OrgWG) im Jahr 2008 die selektivvertragliche Versorgung weiterentwickelt ${ }^{4}$. Nach Ende der Förderung der Integrierten Versorgung nach §140a-d SGB V war das politische Interesse zunehmend auf die hausärztliche Versorgung gerichtet: Seit dem 30. Juni 2009 müssen alle Krankenkassen flächendeckend die hausarztzentrierte Versorgung (HZV)

Dr. med. Dr. sportwiss. H. Giesen, MBA (Univ. of Wales) Geschäftsbereichsleiter Verträge, Vereinigte IKK nach §73b SGB V anbieten. Neben den Effizienzreserven durch strukturelle Veränderungen und einen günstigeren Einkauf ${ }^{5}$ liegen die Optimierungspotenziale der Gesundheitsversorgung übereinstimmend in der intersektoralen Zusammenarbeit ${ }^{6}$. Daher ist es für die Krankenkassen nicht zielführend, sich im Vertragswettbewerb allein auf den hausärztlichen Sektor zu beschränken.

Die notwendigen Rahmenbedingungen für eine Innovationsförderung fehlen im deutschen Gesundheitswesen allerdings vollständig7. Der „Zusatzbeitragsvermeidungswettbewerb" reduziert die Investitionsbereitschaft der Krankenkassen auf ein Minimum ${ }^{8}$. Für Innovationen zur Weiterentwicklung der Vertragsbeziehungen von Krankenkassen und Leistungserbringern oder der solidarisch finanzierten Versorgungssysteme in einer alternden Gesellschaft fehlt der systemische Anreiz ${ }^{9}$.

Die meisten Krankenkassen bleiben daher bis heute strikte Gegner der hausarztzentrierten Versorgung (HZV). Dies ist - bei bisher fehlender Sanktionierung durch die Aufsichtsbehörden - rational, denn:

- Versorgungsmanagement einzuführen, ist komplex und damit finanziell und personell risikobehaftet.

- Die vom Gesetzgeber bisher geduldete verzögerte Umsetzung der HZV ermöglicht im Wettbewerb der Krankenkassen erhebliche Wettbewerbsvorteile. 


\section{THEMA}

- Der Reifegrad der Werkzeuge und die benötigte Führungs- und Managementkompetenz für ein flächendeckendes Versorgungsmanagement ist auch auf Seiten der Leistungserbringer noch gering.

\subsection{Handlungsrahmen einer gesetzlichen Krankenkasse}

Die Förderung der integrierten Versorgung hat vor allem zu regional begrenzten Versorgungsprojekten geführt, wird aber als alternativlos angesehen ${ }^{10}$. Durch den Wegfall der Anschubfinanzierung fehlt den Krankenkassen - mit Ausnahme der Disease-Management-Programme - eine planbare Finanzierungsgrundlage für Selektivverträge. Innovationen müssen aus den Zuweisungen für Leistungen und Verwaltungskosten finanziert werden. Als Konsequenz sinkt die Anzahl der IV-Projekte stetig ${ }^{4}$. Dagegen können flächendeckende Selektivverträge, in die ein relevanter Anteil des Versichertenbestandes eingeschrieben ist, Effizienzen im Gesundheitswesen wettbewerbsrelevant realisieren ${ }^{11}$. Jedoch sind für deren Umsetzung besonders hohe finanzielle Risiken gegeben. Die Umsetzungshindernisse für ein flächendeckendes Versorgungsmanagement dominieren bei kurzfristiger Ausrichtung der Unternehmensstrategie daher deutlich gegenüber den erzielbaren Vorteilen.

Die kurzfristige Refinanzierung von Investitionen in Selektivverträge kann eine Krankenkasse aktuell nur aus Rücklagen oder einem Überschuss der Zuweisungen aus dem Gesundheitsfonds gegenüber den Ausgaben (positiver Deckungsbeiträge) gewährleisten ${ }^{12}$. Vertriebserfolge durch Akquise positiver Deckungsbeiträge werden durch die Vertriebskosten meist erst im zweiten Mitgliedsjahr relevant. Eine Kostenreduktion durch effektive Steuerung sowie die Vermeidung von Folgekosten durch Prävention sind erst mittel- und langfristig wirksam ${ }^{13}$. Somit ist es nicht verwunderlich, dass die Interessen der Krankenkassen vorwiegend auf Kostenmanagement ${ }^{14}$ und die Optimierung der Zuweisung aus dem Gesundheitsfonds ausgerichtet waren und noch stets sind ${ }^{15}$. Obgleich die Optimierung der Morbi-RSA-Zuweisung keine planbare Komponente eines Geschäftsmodells für Selektivverträge darstellen kann ${ }^{16}$, wird sie mit diesen besonders in Verbindung gebracht. Dagegen ist die Einnahmesicherung einer Krankenkasse nicht allein durch Selektivverträge zu erreichen ${ }^{17}$. Aktuell zeigt sich ein Rückgang der Morbi-RSA-Zuweisungen in einzelnen hierarchisierten Morbiditätsgruppen (HMG), die auf eine relevante Veränderung in der DiagnoseKodierung hinweisen, ohne dass bereits flächendeckend Selektivverträge bestehen. Die strikte Sanktionierung von Manipulationen der Diagnose-Kodierung ist daher richtig und wichtig ${ }^{18}$. Dies gilt sowohl für Veränderungen der Diagnose-Häufigkeit aus Selektivverträgen, wenn Anreize durch die Vergütungssystematik gegeben sind, als auch für sonstige Auffälligkeiten, für die keine nachvollziehbare Begründung geliefert werden kann.

Demgegenüber steht die Verbesserung der Kodier-Qualität in Selektivverträgen als Ergebnis der Optimierung der Versorgungssteuerung und der Behandlungsqualität. Es wird für die Aufsichtsbehörden eine wichtige Aufgabe sein, medizinisch begründete Veränderungen der DiagnoseHäufigkeit von manipulativen zu unterscheiden ${ }^{16}$. Gelingt dies nicht, ist ein weiterer systemischer Anreiz gegen das aktive Versorgungsmanagement und für die kurzfristige Einnahmesicherung bei den Krankenkassen gesetzt.

\subsection{Sind Versorgungsmanagement und die aktive Versichertensteuerung politisch gewünscht?}

Die aktuelle Regierung fordert wie auch schon deren Vorgängerkoalitionen Wettbewerb im Gesundheitswesen. Doch sind seit dem GKV-Wettbewerbsstärkungsgesetz (GKV-WSG) bis zur aktuellen Version des GKV-Finanzierungsgesetz (GKV-FinG) die Anreize für einen Wettbewerb im Gesundheitswesen wirklich gesetzt worden? Der Umsetzung der hausarztzentrierten Versorgung stehen offene datenschutzrechtliche Fragen ${ }^{19}$ und wirtschaftliche Nachteile für Krankenkassen, die die Vorgaben des §73b SGB $\mathrm{V}$ befolgt haben, gegenüber. Die Krankenkassen, die eine seit dem 30.06.2009 verpflichtende HZV umsetzen, haben Mehrkosten in zusatzbeitragsrelevanter Höhe. Diese gliedern sich in Mehrausgaben für Programmkosten und Leistungsausgaben. Der Verwaltungsaufwand der inhaltlich frei verhandelbaren Selektivverträge ( $\$ 73 b, 73 c, 140 a-d$ SGB V sowie Modellvorhaben und Strukturverträge) ist vergleichbar mit den Disease-Management-Programmen. Hier lag die Programmkostenpauschale in den Anfangsjahren bei 55 Euro pro Versichertenteilnahmejahr und liegt aktuell bei 36 Euro pro Versichertenteilnahmejahr ${ }^{20}$. Über die Mehrkosten für Leistungsausgaben der HZV besteht aktuell keine Transparenz. Presseinformationen zufolge liegt der durchschnittliche Wert bei etwa 76 Euro pro Teilnehmer jährlich ${ }^{21}$. Da die Zuweisungen aus dem Gesundheitsfonds jedoch für „gesetzestreue“ Krankenkassen nicht anders sind als für Krankenkassen, die die Verpflichtung zur HZV nicht umsetzen, entsteht daraus ein dauerhafter Wettbewerbsnachteil. Unterstellt man eine (ambitionierte) „interne Kompensation“ der Verwaltungskostenausgaben von 31 Euro durch gesetzestreue Krankenkassen, so resultiert (76 + 55 - 31 Euro) eine Mehrbelastung von 100 Euro pro teilnehmendem Versicherten pro Jahr (dies entspricht etwa der Höhe des maximalen pauschalen Zusatzbeitrags von 8 Euro pro Monat). Nimmt man eine durchschnittliche bundesweite Teilnahmequote pro Jahr in Höhe von 25\% $\%^{22}$ an, so resultieren je Versicherten Mehrkosten in Höhe von 25 Euro pro Jahr, die Krankenkassen ohne HZV-Vertrag unsanktioniert als Wettbewerbsvorteil erzielen.

Dieser Wettbewerbsnachteil wird dadurch verstärkt, dass außerhalb von Bayern und Baden-Württemberg datenschutzrechtliche Bedenken zu einem Imageschaden der „gesetzestreuen“ Krankenkassen führen ${ }^{23}$. Wie soll einem Versicherten und der Presse plausibel gemacht werden, dass die HZV zwar seit 15 Monaten gesetzlich mit den kontrahierten Vertragspartnern vorgeschrieben ist, die Verschiebung des Echtbetriebs der HZV aus ungeklärten datenschutzrechtlichen Fragen in Bezug auf diese Vertragspartner aber trotzdem notwendig ist? 


\section{Optionen für einen Versorgungswettbewerb - Versorgungsmanagementpauschale}

Erheben Krankenkassen einen Zusatzbeitrag, führt das zu erheblichen Mitgliederverlusten ${ }^{24}$. Eine planbare und wettbewerbsneutrale Finanzierung der Kosten von Selektivverträgen ist für die Krankenkassen im „Zusatzbeitragsvermeidungswettbewerb" daher sehr bedeutsam.

\subsection{Zuweisung aus dem Gesundheitsfonds analog DMP- Pauschale}

Die Zuweisung einer DMP-Pauschale je nachgewiesenem Versichertenteilnahmejahr hat die Existenz der DMPProgramme gesichert. Diese sind heute ein international anerkannter Weg des Versorgungsmanagements ${ }^{25}$. Diese Pauschale könnte um eine weitere Pauschale gleicher Art der Umsetzung ergänzt oder zu einer einzigen Versorgungsmanagementprogramm-Pauschale (VMP-Pauschale) zusammengefasst werden. Kassen mit Selektivverträgen, die den Nachweis der Wirtschaftlichkeit ex ante erbracht haben bzw. für HZV-Bestandsverträge den Wirtschaftlichkeitsnachweis ex post erbringen müssen, könnten als Ausgleich für die finanzielle Mehrbelastung die Zuweisung einer VMP-Pauschale je Versichertenteilnahmejahr aus dem Gesundheitsfonds erhalten. Die VMP-Pauschale mindert analog der DMP-Pauschale die über den Fonds verteilbaren Mittel. Analog der Regelung zur DMP-Pauschale muss als Grundlage für die Zuweisung aus dem Fonds die Anzahl der wirksam eingeschriebenen Versicherten von den Kassen nachgewiesen werden. Regelhafte Prüfungen durch die Aufsicht sind zu implementieren.

\subsection{Verpflichtender Wirtschaftlichkeitsnachweis}

Selektivverträge sollen die Wirtschaftlichkeit der Versorgung verbessern. Die Verpflichtung zum Nachweis der Wirtschaftlichkeit für bestehende und zukünftige Selektivverträge, für die je teilnehmenden Versicherten eine Zuweisung aus dem Gesundheitsfonds erfolgen soll, ist im vorliegendem Gesetzesentwurf des GKV-FinG somit zu begrüßen. Die Methodik des Wirtschaftlichkeitsnachweises sollte verbindlich und für alle Versorgungsprogramme in gleicher Weise vorgeschrieben und einheitlich bei den Aufsichtsbehörden überprüft werden.

\subsection{Pauschale für durchschnittliche Mehrkosten der Leistungsausgaben als Anschubfinanzierung für Selektivverträge}

Analog der Regelung im DMP sollte der Gesetzgeber die durchschnittlichen Kosten für ambulante ärztliche Leistungen ermitteln und als einheitliche Pauschale je teilnehmenden Versicherten auszahlen. Aufgrund der nachzuweisenden Wirtschaftlichkeit der HZV-Verträge stellt die Pauschale für ärztliche Leistungen über die Zeit eine Art Anschubfinanzierung für die Konzeptentwicklung und Pilotierung von sektorenübergreifenden Versorgungspro- grammen dar. Die mittelfristig erzielten Effizienzgewinne können zur Investition in weitere Versorgungsprogramme dienen. Alternativ kann die Pauschale für ärztliche Leistungen deshalb auch zeitlich begrenzt werden.

\subsection{VMP-Pauschale unterstützt politisches Ziel des Versorgungswettbewerbs}

Je nach Höhe der Zuweisung der VMP-Pauschale bleibt der Anreiz für eine flächendeckende selektivvertragliche Versorgung bestehen, so dass Krankenkassen über innovative Verträge Effizienz und Effektivität der Versorgung aktiv verbessern können. Wünscht der Gesetzgeber den Abschluss weiterer Hausarztverträge und ergänzender Selektivverträge bzw. relevante Einschreibungen von Versicherten in Selektivverträge/ Versorgungswahltarife nach \$53 (3) SGB V, so sollte die VMP-Pauschale höher festgesetzt werden. Im Jahr 2011 beträgt die DMP-Pauschale 168 Euro pro Versichertenteilnahmejahr ${ }^{20}$. Je nach politischer Zielsetzung könnte eine einzige VMP-Pauschale zwischen den tatsächlich nachgewiesenen Mehrkosten aller Selektivverträge und der aktuellen DMP-Pauschale festgelegt werden. Da durch den „Vorwegabzug“ der Versorgungsmanagement-Pauschale der Gesundheitsfonds für die weiteren Zuweisungen anteilig unterdeckt sein wird, kann keine Krankenkasse mittelfristig ohne Teilnahme am Versorgungswettbewerb ohne Zusatzbeitrag bestehen bleiben.

\section{Patientenbedürfnisse im Mittelpunkt statt Patient als Mittel}

Über eine finanzielle Planungsgrundlage für Selektivverträge können Kassen differenzierte Versorgungsprodukte entwickeln, erproben und flächendeckend anbieten. Zielgruppen, die bisher für Präventionsangebote kaum erreichbar waren, sind durch die Krankenkasse zu 100\% ansprechbar, sofern die vorliegenden Routinedaten zur Analyse verwendet werden dürfen. Mit finanziellen Anreizen sind auch schwer erreichbare Zielgruppen motivierbar ${ }^{26} .78 \%$ der Bürger sehen die Verantwortung für ihre Gesundheit in eigenen Händen, 89\% wünschen sich Informationen über die Qualität von Leistungserbringern, $50 \%$ sehen zu viel Ineffizienz und Bürokratie im deutschen Gesundheitswesen und eine deutliche Mehrheit hat Befürchtungen hinsichtlich Speicherung und Weitergabe von Patientendaten ${ }^{27}$. Daher sollten auch die datenschutzrechtlichen Bedingungen für eine erfolgreiche Umsetzung des zielgruppenspezifischen Versorgungsmanagements eindeutig geregelt werden. Eine Anpassung des §284 SGB V sollte die Verwendung der vorliegenden Abrechnungsdaten für die Vorbereitung, Durchführung und Steuerung von Selektivverträgen eindeutig erlauben. Auf Basis der vorliegenden Routinedaten können Krankenkassen zielgruppenspezifisch Informationsmaterialien anbieten, evidenzbasierte Patienteninformationen anlassbezogen bereitstellen und damit ihren Anteil zur partizipativen Entscheidungsfindung und Transparenz der Versorgungsqualität in Zusammenarbeit mit dem niedergelassenen Arzt leisten ${ }^{28}$. 
Im ersten Halbjahr 2011 soll das „Versorgungsgesetz“ die Gesundheitsversorgung im ländlichen Bereich verbessern und die Honorierung der Ärzte reformieren ${ }^{29}$. Über das Versorgungsgesetz könnte der Gestaltungsspielraum für flächendeckende selektivvertragliche Versorgungskonzepte geschaffen werden. Die VMP-Pauschale würde den Kassen eine solide Kalkulationsgrundlage bieten, um mit innovativen Versorgungsformen Wettbewerbsvorteile durch konsequente Erfüllung der Kundenbedürfnisse zu erzielen. In Abhängigkeit von der Höhe der VMP-Programmkostenpauschale wird die Innovationsbereitschaft der Krankenkassen mehr oder weniger stark gefördert. Ein „Einschreibewettbewerb“ in die selektivvertragliche Versorgung wäre somit als positiver Effekt einer gut ausgestalteten VMPPauschale möglich und sinnvoll, da die Wirtschaftlichkeit der Versorgung zwingend nachgewiesen werden muss. Letzteres muss daher in jedem Fall ergänzender Bestandteil der Einführung einer VMP-Pauschale sein. Die Sanktionierung einer unwirtschaftlichen Mittelverwendung sollte vorab definiert und deren Umsetzung verpflichtend sein. Unter diesen Bedingungen könnten sinnvolle Innovationsanreize geschaffen, verdeckte Rationierung und kostentreibende Anreizsysteme ${ }^{30}$ beseitigt sowie qualitätsorientierte Vergütungssysteme ${ }^{31}$ erprobt und weiterentwickelt werden.

\section{Fußnoten:}

1 Interview Philipp Rösler: www.liberale.de/Mehr-Wettbewer-mehr-Freiheit-weniger-Buerokratie/5802c10289i1p69/index.html; 11.08.2010

2 Sachverständigenrat zur Begutachtung der Entwicklung im Gesundheitswesen: Gutachten 2000/2001, 2003, 2005, 2007, Sondergutachten 2009.

3 Cassel . D./I. Ebsen/S. Greß/K. Jacobs/S. Schulze/J. Wasem (2008). Vertragswettbewerb in der GKV - Möglichkeiten und Grenzen vor und nach der Gesundheitsreform der Großen Koalition. www.wido.de; Suchbegriff: „Vertragswettbewerb“.

4 Ballast, T. (2010). Selektivverträge in der vertragsärztlichen Versorgung. In: Rebscher H. /S. Kaufmann (Hrsg.): Innovationsmanagement in Gesundheitssystemen. Heidelberg, medhochzwei: 255-270.

5 Augurzky B./H. Tauchmann/A. Werblow/S. Felder (2009). Effizienzreserven im Gesundheitswesen. RWI Materialien, Heft 49. www.rwi-essen.de/ publikationen/rwi-materialien/13/

6 Zerth, J. (2010). Dimensionen von Innovation: Ökonomische Aspekte im Kassenwettbewerb. In: Rebscher, H. /S. Kaufmann (Hrsg.): Innovationsmanagement in Gesundheitssystemen. Heidelberg, medhochzwei: 3-18

7 Stuppardt, R. (2010). Innovationsförderung durch politische Reformprozesse - die deutsche Perspektive. In: Rebscher, H. /S. Kaufmann (Hrsg.): Innovationsmanagement in Gesundheitssystemen. Heidelberg, medhochzwei: 3-18

8 S. Greß/K. Jacobs/S. Schulze (2010). GKV-Finanzierungsreform: Schwarzgelbe Irrewege statt gezielter Problemlösung. Gesundheits- und Sozialpolitik 64 (4): 16-29

9 Bormann, R./E. Fiedler/C. Herrmann/F. Knieps/P. König/A. Lang/G. Naegele/M. Reichert/H. Rothgang/D. Schaeffe (2009). Zukunft des Gesundheitssystems - Solidarisch finanzierte Versorgungssysteme in einer alternden Gesellschaft. Friedrich-Ebert-Stiftung. WISO Diskurs. www. fes.de/wiso

10 Osterloh, F./S. Rieser (2010): Interview mit Prof. Dr. Volker Amelung: Zur integrativen Versorgung gibt es keine Alternative. Dtsch. Arztebl 107 (17): A-791-794
11 Öffentliche Anhörung des Ausschusses für Gesundheit des Deutschen Bundestages am 25.10.2010 in Berlin. Schriftliche Stellungnahme von Dr. Christopher Hermman zum GKV-FinG. www.bundestag. de/bundestag/ausschuesse17/a14/anhoerungen/d_GKV-FinG/ Stellungnahmen/17_14_0074_47_.pdf

12 Eble, S. (2010). Versorgungsmanagement - zukünftiges Geschäftsfeld der Industrie? Die Krankenversicherung 9: 269-275

13 Lüngen, B./K. Liese (2010). Innovation im Versorgungsmanagement am Beispiel der Hochkostenfälle. In: Rebscher, H. /S. Kaufmann (Hrsg.): Innovationsmanagement in Gesundheitssystemen. Heidelberg, medhochzwei: 3-18

14 Nebling, T. (2009): Gesundheitsfonds und morbiditätsorientierter Risikostrukturausgleich als zielkonforme Rahmenbedingung für die GKV? In: Göpffarth, D./S. Greß/ K.. Jacobs/J. Wasem (Hrsg.): Jahrbuch Risikostrukturausgleich 2008: Morbi-RSA. Sankt Augustin, Asgard: 213 237.

15 Arndt, V./L. Tennie/D. Göpffarth (2010): Anstieg spezifischer ambulanter Diagnosestellungen nach Bekanntgabe der im Morbi-RSA 2009 berücksichtigten Diagnosen und Aufgreifkriterien. GMDS Jahrestagung 2010. www.gmds2010.de/cms/wordpress/wp-content/uploads/ folien/250.pdf

16 Dudey, S. (2010). Optimale Morbiditätsabbildung bei fehlerhaften und unvollständigen Datengrundlagen? In: Göpffarth, D./S. Greß/ K.. Jacobs/J. Wasem (Hrsg.): Jahrbuch Risikostrukturausgleich 2009/2010: Von der Selektion zur Manipulation. Heidelberg, medhochzwei: 27-39.

17 Dieckmann, R. (2009). Aspekte neuer Vertragsstrategien der Krankenkassen aus Sicht der DAK. Die Krankenversicherung 10: S. 266-269.

18 Gesetzesänderung mit der 15. AMG-Novelle räumt dem BVA Überprüfungs- und Sanktionsbefugnisse ein; s.a http://www.bgbl.de/Xaver/start. xav?startbk=Bundesanzeiger_BGBl Suchbegriff: 2009 Teil I Nr. 43, Seite 1990 und http://www.gesundheit-adhoc.de/index.php?m=1\&id=6596

19 Weichert, T.: https://www.datenschutzzentrum.de/medizin/gkv/hausarztzentrierte-versorgung2.html; 11.02.2010

20 Pfeiffer, D. (2010): Bestimmung des GKV-Spitzenverbandes nach § 38 Abs. 2 RSAV über die Höhe der DMP-Programmkostenpauschale und das Nähere zum Meldeverfahren für in DMP eingeschriebene Versicherte. Schreiben des GKV-Spitzenverband der Krankenkassen an den Präsidenten des Bundesversicherungsamtes vom 22.09.2010

21 Der Gelbe Dienst 14/2010; als Primärquelle wird das Bundesministerium für Gesundheit genannt.

22 Quote HZV-Vertrag der AOK Bayern: 55\% zum Ende des 2. Abrechnungsquartals gem. Rundfax Hausärzteverband Bayern

23 Dienst für Gesellschaftspolitik 41-10, siehe auch www.aerzteblatt.de/v4/ news.asp?id=42926

24 Dienst für Gesellschaftspolitik 30-10, siehe auch Mühlnikel, I. (2010): Kassenwettbewerb - Schreckgespenst. kma 10: 22-27

25 Miksch A. /G. Laux/D. Ose/S. Joos/S. Campbell/B. Riens/J. Szecsenyi (2010). Is there a survival benefit within a German primary care-based disease management program? Am J Manag Care. 16(1): 49-54.

26 Katte J./K. Neumann (2009). Strukturen der Prävention. Roland Berger Strategy Consultants. www.rolandberger.com; Suchbegriff: Structures of prevention.

27 Accenture Citizens Experience Studie (2010). Beiträge zur Gesellschaftspolitik 8-10 vom 21.10.2010

28 Donner-Banzhoff, N. (2011). Partizipative Entscheidungsfindung und Patientenschulung. In: Rebscher H. /S. Kaufmann (Hrsg.): Innovationsmanagement in Gesundheitssystemen. Heidelberg, medhochzwei: 64-67

29 KBV kompakt vom 20.10.2010

30 Wehling, M. (2009). Das Geld im Gesundheitswesen reicht noch lange, wenn die Verschwendung aufhört. Monitor Versorgungsforschung 6: 46-51

31 Mansky, T. (2010). Qualitätsorientierte Vergütung: Was wäre machbar? Der Chirurg BDC 1: 6-9 